\title{
Brain Connectivity and Applications to Neuropsychology: Introduction to the Special Issue of Neuropsychology Review
}

\author{
Bonnie J. Nagel
}

Received: 30 January 2014 / Accepted: 4 February 2014 / Published online: 13 February 2014

(C) Springer Science+Business Media New York 2014

In recent years, understanding of human brain functioning has dramatically been enhanced by neuroimaging techniques. While the field of neuropsychology was once largely informed by postmortem and lesion studies, which provided insight into specific focal brain and behavioral relationships, it is now better appreciated that regions of the brain do not operate in isolation but rather function as parts of rich integrated networks. The work that has most recently contributed to our understanding of network level brain functioning is that using analytic techniques to exploit temporal correlations in the blood oxygen level-dependent (BOLD) response across the brain, otherwise known as functional connectivity. This analysis can be performed while engaged in a task or while at rest. The latter is less bound by the limitations imposed by traditional task-based functional magnetic resonance imaging (fMRI) studies, in that it does not rely on a particular level or comparability of task performance and is relatively easy and fast to collect. The amenability of the resting state paradigm to creative study of a variety of patient populations, even those with dementing disorders, has enabled unprecedented expansion of knowledge regarding the underlying neurobiology of many disease states.

\section{In This Issue}

The authors of this special issue detail how functional connectivity studies have advanced our understanding of the brain as it relates to selective aspects of neuropsychological functioning in normal development and aging, disease states, and

\section{B. J. Nagel $(\bowtie)$}

Departments of Psychiatry and Behavioral Neuroscience, Oregon

Health \& Science University, 3181 SW Sam Jackson Park Road, DC7P, Portland, OR 97239, USA

e-mail: nagelb@ohsu.edu with treatment. We start from an historical perspective by directing the reader to the pioneering work of D. O. Hebb and his book, The Organization of Behavior, published in 1949 [http://krishikosh.egranth.ac.in/bitstream/1/2027513/1/ HS1199.pdf; http://deeplearning.cs.cmu.edu/pdfs/Hebb. 1949.pdf]. Therein are the theoretical origins of brain connectivity, described in chapter 4, "The First Stage of Perception: Growth of the Assembly." The noting of this chapter sets the stage for the empirical reviews, as Hebb describes the notion of cell "assembly," forming the basis for what is now considered functional connectivity. With this formative chapter, and through the five empirical review articles, this special issue aims to demonstrate the utility of functional connectivity studies for uncovering the neurobiological underpinnings of disease and behavior, as well as how these techniques could be used to identify neurobiological markers of rehabilitation. In addition to reviewing how functional connectivity studies have been employed to understand a specific patient population or phenomenon in neuropsychology, each review highlights key methodological challenges for using this technique to study the connectivity of the human brain. Such issues include addressing nuisance signal in the data, pros and cons of seed-based versus independent component analysis approaches, whole-brain versus region-of-interest analyses, and analytic strategies to address network properties as opposed to simply one-to-one connections (e.g., graph theory). Despite the preponderance of methodological considerations that constitute this type of investigation, the reviews presented here emphasize the promise of this technique to enhance understanding of brain and behavior relationships across the lifespan in health and disease.

Park, Wang, and Posner introduce this issue with their review on how functional connectivity studies have informed our understanding of the neuropathological bases for attention deficit/hyperactivity disorder (ADHD). Findings from these 
studies converge on three distinct networks in the brain: the default mode network (DMN), active during rest and often suppressed during active tasks; a cognitive control network; and both cognitive and affective cortico-striato-thalamo-cortical circuitry. Overall, these studies suggest a lack of typically observed functional segregation between the DMN and cognitive control networks in ADHD, reduced withinDMN connectivity, and atypical connectivity in both cognitive and affective cortico-striato-thalamo-cortical loops, the latter of which shows dissociable correlates with ADHD symptomatology.

Continuing studies on neurodevelopment, Maximo, Cadena, and Kana next explore functional connectivity studies of autism. With the ultimate goal of using functional connectivity to identify neurobiological underpinnings of autism, and therefore possibly aid diagnosis, studies have demonstrated both hypo- and hyper-connectivity in several networks, with findings of both more short-distance connectivity and attenuated long-distance connectivity across the autistic brain. In addition, Maximo and colleagues detail studies showing parallel aberrations in the structural connectivity of the autistic brain, suggesting that the atypical connectivity of the brain may be a pathophysiological feature in the neurobiology of autism.

Moving into adult populations with a review on brain network abnormalities in schizophrenia and a comprehensive overview of analytic techniques, van den Heuvel and Fornito detail studies of both structural and functional connectivity in schizophrenia, as well as sophisticated graph theory approaches designed to assess features of network integrity. As is the case with the preceding reviewed populations, schizophrenia is associated with widespread connectivity abnormalities throughout the brain, including atypical connectivity in DMN, cognitive control, salience, and fronto-limbic networks. Whereas structural connectivity studies have largely demonstrated attenuated neuroanatomical connectivity in schizophrenia, functional connectivity studies have shown evidence for both hypo- and hyper-connectivity, with frontal brain regions most commonly implicated. At a network level, both positive and negative schizophrenic symptoms have been associated with lower global network efficiency and less clustering in the brain, with lower connectivity related to poorer cognitive functioning.

Dennis and Thompson address later stages of the lifespan in their contribution highlighting changes in functional connectivity in both normal and pathological aging, including Alzheimer's Disease. To examine resting state functional connectivity in the brain during normal aging and Alzheimer's Disease, Dennis and Thompson focus on seed-based, independent components analysis (ICA), and graph theory techniques. Not surprisingly, functional connectivity in the brain declines over the normal aging process with regional variability, and abnormally fast degradation of connections in the Alzheimer's Disease state. As is the case with many disorders, one of the networks most implicated in this disease process is the DMN. Strikingly, while not the focus of the review, the authors also detail findings from positron emission tomography (PET) studies showing that regions of the DMN are susceptible to amyloid plaque deposition, which may be contributory in the decline of functional connectivity of this network.

The final review by Kelly and Castellanos describes how these neuroimaging methods can be used to study neural plasticity and recovery more broadly. They note that functional connectivity is modified by experience and that both practice and training highlight the plasticity of brain network integrity. This phenomenon, of particular importance to the field of neuropsychology as it can help to explain the neurobiology of brain injury and rehabilitation, can be observed following training on perceptual, motor, and high level cognitive tasks. These effects have been observed in numerous brain networks, and likely reflect increases in network efficiency, as well as reorganization and compensation of brain processes.

\section{Concluding Comments}

As is evident from the work put forth in this special issue, functional connectivity studies have vastly advanced our knowledge on the neurobiology of development, aging, and disease and how the brain responds, at a network level, to experience. Additional work is needed to learn more about the behavioral and neuropsychological correlates of connectivity patterns in the brain and to specify how different disease states are overlapping and dissociable from one another (i.e., conducting studies across diagnostic categories and continua). One outcome of these pursuits will be to extend the application of functional connectivity studies to neuropsychological assessment and to ground neuroimaging findings in ecologically valid contexts. Even in their infancy, it is clear that methods for discovering functional connectivity hold promise for informing the field of neuropsychology, both at the diagnostic and treatment level. 